Perspectivas ISSN: 2590-9215 Volumen 3 (2) Julio - Diciembre de 2018, páginas 17-33

\title{
Análisis de la movilidad de docentes universitarios del Táchira dentro de la complejidad de los escenarios contemporáneos. (Estudio de caso de la Universidad Nacional Experimental del Táchira) \\ Context of the mobility of Venezuelans within the complexity of contemporary scenarios \\ Contexto da mobilidade dos venezuelanos dentro da complexidade dos cenários contemporâneos
}

\footnotetext{
Ender José Barrientos-Monsalve ${ }^{\mathrm{a} *}$, Juan Carlos Garmendia-Mora ${ }^{\mathrm{b}}$, Carolina Marulanda-Ascanio ${ }^{\mathrm{c}}$, Nury Angelica Rosales Nuñez ${ }^{\mathrm{d}}$

a* Doctor en Ciencias Gerenciales, dr.ender.utel@gmail.com, ORCID 0000-0001-6673-0223, Fundación de Estudios Superiores Comfanorte, Cúcuta, Colombia. boctor en ciencias Gerenciales, juangarmendia@yahoo.com, ORCID 0000-0003-0127-4292, Universidad Nacional Experimental Politécnica de la Fuerza Armada Nacional, Táchira, Venezuela. ${ }^{\mathbf{c} E s p e c i a l i s t a ~ e n ~ G e r e n c i a ~ F i n a n c i e r a, ~ c \_m a r u l a n d a @ f e s c . e d u . c o, ~ O R C I D ~ 0000-0002-1953-8850, ~}$

Fundación de Estudios Superiores Comfanorte, Cúcuta, Colombia. ${ }^{\mathbf{d}}$ Magister en orientación de la conducta, nuryrunica@gmail.com, ORCID 0000-00021361-770X, Universidad Cecilio Acosta, San Cristóbal, Venezuela.
}

Forma de citar: Barrientos, E. J., Garmendia, J.C., Marulanda, C. \& Rosales, N.A. (2018). Análisis de la áneosmovilidad de docentes universitarios del Táchira dentro de la complejidad de los escenarios contemporáneos.

(Estudio del caso de la Universidad Nacional Experimental del Táchira). Perspectivas, 3(2), 17-33.

Recibido: Enero 20 de 2018

Aceptado: Junio 01 de 2018

Palabras clave

Contemporaneidad

Docentes

Movilidad

Universidad
Resumen: El propósito de este estudio, fue analizar la emigración de los docentes venezolanos durante el Periodo del 2015 al 2017, dentro de un caso de estudio en particular como el de la Universidad Nacional Experimental del Táchira, y su relación con la crisis socio política que vive el país en el marco contemporáneo, ante esto se trabajó bajo el objetivo de Analizar la movilidad de docentes universitarios de la universidad Experimental del Táchira dentro de la complejidad de los escenarios contemporáneos. Es por ello que se tomó como muestra unos informantes claves que fueron profesores universitarios de esa casa de estudios superiores, siendo personas que han salido a diferentes países se les aplicó una entrevista abierta para ver y describir porque salieron bajo que motivación y hacia dónde y por qué escogieron esos sitios de destino para seguir con sus vidas con la familia. La investigación tuvo un enfoque cualitativo descriptivo ya que se describió las experiencia

\footnotetext{
*Autor para correspondencia

Ender José Barrientos Monsalve

dr.ender.utel@gmail.com
}

https://doi.org/10.22463/25909215.1583

2590-9215 2017 Universidad Francisco de Paula Santander. Este es un artículo bajo la licencia CCBY 


\section{Keywords}

Contemporaneity

Teachers

Mobility

University

\section{Palavras chave}

Ensino médio

Estudantes

Avaliação

Escolas

Provas

Resultados de cada docente entrevistado para que cada uno diera su experiencia hacia la investigación, en esto esta investigación tuvo como conclusión basada en las descripciones de los docentes que la salida de Venezuela se debió a causa de las situaciones principalmente económica por no cubrir los gastos necesarios para poder vivir tranquilamente, seguidamente las otras categorías que salieron fueron la de la seguridad y políticas económicas afectando con esto toda la dimensión del entorno familiar para poder vivir humana mente bien.

Abstract: The purpose of this study was to analyze the emigration of Venezuelan teachers during the period from 2015 to 2017, within a case study in particular as the National Experimental University of Táchira, and its relationship with the socio-political crisis that lives the country in the contemporary framework, before this was worked under the objective of analyzing the mobility of university teachers of the Experimental University of Táchira within the complexity of contemporary scenarios. That is why it was taken as a sample key informants who were university professors from that house of higher education, being people who have left to different countries they are applied an open interview to see and describe because leaving you under what motivation and where and why who chose those destinations to continue their lives with the family. The research had a qualitative descriptive approach since the experience of each teacher interviewed was described so that each one would give their experience to the investigation, in this this investigation had as a conclusion based on the descriptions of the teachers that the exit from Venezuela was due to cause of the situations mainly economic for not covering the necessary expenses to be able to live quietly, next the other categories that left were the one of the security and economic policies affecting with this all the dimension of the familiar surroundings to be able to live humanly well.

Resumo: O objetivo deste estudo foi analisar a emigração de professores venezuelanos durante o período de 2015 a 2017, dentro de um estudo de caso particular, como o da Universidade Nacional Experimental de Táchira, e sua relação com a crise sócio-política que o país está experimentando no quadro contemporâneo, com o objetivo de analisar a mobilidade dos professores universitários da Universidade Experimental de Táchira dentro da complexidade dos cenários contemporâneos. É por isso que tomamos como amostra alguns informantes chave que eram professores universitários desta universidade, sendo pessoas que saíram para diferentes países, uma entrevista aberta foi aplicada a eles para ver e descrever porque saíram com que motivação, onde e porque escolheram esses lugares de destino para continuar com suas vidas com a 
família. A pesquisa teve uma abordagem descritiva qualitativa, uma vez que a experiência de cada professor entrevistado foi descrito para que cada um pudesse dar a sua experiência para a pesquisa. Esta pesquisa teve como conclusão com base nas descrições dos professores que a partida da Venezuela foi devido principalmente a situações econômicas, porque eles não cobrir as despesas necessárias para poder viver em paz, então as outras categorias que saíram foram as de segurança e políticas econômicas, afetando com isso toda a dimensão do ambiente familiar para ser capaz de viver a mente humana bem.

\section{Introducción}

La movilidad humana ha existido desde los inicios de la humanidad siendo generada por diversos motivos que anteceden a la cobertura de las necesidades mínimas necesarias para la subsistencia humana, entre las que se pueden comentar estabilidad alimentaria la cual debe establecer las condiciones necesarias para encontrar la alimentación de las personas integrantes de la familia, así mismo poder vivir en ambientes adecuados, con base a estas características se puede enfocar y ver la movilidad, presentándose cuando estas necesidades empiezan a no tener sus coberturas básicas, ya sea por situaciones climáticas u otro tipo de consecuencia, y esto hace que los miembros de una comunidad enfrenten situaciones adversas que no las deja vivir armónicamente, y es de allí donde se toma la decisión de trasladarse a otro lado buscando mejores condiciones de vida, enfrentando otros contextos como culturales, políticos, sociales y prácticamente, es por ello que se inicia la movilidad humana, y esta situación se ha mantenido hasta la actualidad en donde la emigración dentro de los escenarios sociales se basa en la búsqueda de mejores condiciones de vida que puedan igualar o mejorar dichas condiciones.

De esta manera como se presenta el fenómeno migratorio algunas de las conceptualizaciones que señalan son: Gómez (2010) establece:

"La migración ha sido una actividad consustancial a la vida del hombre y le ha acompañado a lo largo de su historia. La conformación y estructuración de los pueblos ha obedecido en gran parte a migraciones; por lo tanto, hay que mirar sus causas y efectos desprovistos de prejuicios y pretensiones perversas" (p. 84).

En este sentido sabiendo que esto es una situación especial de las personas y los pueblos en gran parte se han formado basados migraciones y emigraciones, el mismo autor señala que la migración internacional es el desplazamiento de personas de un país a otro para ejercer su residencia, conformando así pueblos y ciudades fomentando la interculturalidad.

Además esta situación migratoria se presenta en varias regiones del mundo y específicamente en Latinoamérica donde evoluciona existiendo movilidades no solo para países vecinos sino para contextos o regiones mucho más alejadas dentro del continente, al respecto Asakura (2014), señala:

"La migración centroamericana, antes de los años setenta del siglo XX, tenía un predominante carácter interregional, es decir, los movimientos poblacionales se daban de un país a otro en la misma región centroamericana" (p. 14).

Así mismo para Gómez (2010), establece las causas sobre la migración internacional estableciendo las socioeconómicas, políticas, psicológicas, culturales y propias de la condición humana. En este orden de ideas del autor, las causas económicas siendo una de las más predominantes en estos grupos sociales empiezan a originar por la diferencia de ingresos económicos que se presentan 
entre los países, así como la manera de adquisición de bienes y servicios del contexto económico del país receptor, y algo muy importante la demanda de empleos que ofrecen una estabilidad a las familias que llegar a territorios nuevos.

Dentro del marco de las consecuencias de las migraciones, las situaciones jurídicas juegan un estado primordial por cuanto la normativa que prohíbe las importaciones y exportaciones a nivel comercial afectando a pueblos que no cuentan con una legislación que les permita tener una economía basada en la defensa jurídica y protección a los inversionistas, incidiendo de manera directa la sociedad al no llegarle productor de primera necesidad que no son fabricados en los países donde se origina la emigración.

Ante esto se empieza a presentar las motivaciones para emigrar, incidiendo entro de los escenarios sobre la salud y la psicología de las personas, y así lo señala Gómez (2010), en donde:

"Determinados por fenómenos de estrés y motivación, ya que ciertas actividades no motivan, tienen baja reputación y no brindan ascenso social a los nativos de los países de destino de la migración" (p.85).

Así mismo el citado autor establece que las causas médicas más que todo en personas mayores no cuentan con acceso a la salud y por consiguiente para seguir viviendo deben buscar otros horizontes para poder ser atendidos y estar estable en sus patologías.

Este contexto complejo sobre la migración que se detalló anteriormente apuntando algunas causas más significativas, también se refleja en los países de llegada de donde se evidencia los movimientos migratorios, por cuanto los países receptores pasan por este fenómeno cuando no están preparados para ello afectando y cambiando sus contextos para poder estabilizar y dar un equilibrio con las personas que llegan como con las que son nativas de ese país, para Gómez (2010):

"Este tratamiento está plagado de muchos prejuicios infundados que son explotados en el concierto electoral, esgrimiendo comentarios sobre la inmigración como causante del aumento del desempleo, caída de los salarios, disputa de los servicios sociales, deprimir los precios de bienes muebles e inmuebles en sus lugares de residencia e infestar de plagas y enfermedades su entorno, como también el de generar violencia y delincuencia" (p.86).

En este sentido se puede evidenciar la complejidad de este escenario afectando a todos los involucrados dentro de sus dimensiones particulares, ya que los países receptores deben formularse políticas para poder recibir esa gran cantidad de personas que de algún modo pueden estar señaladas dentro de los términos de migrantes o refugiados por las situaciones que se viven en sus países de origen en no brindarles las situaciones mínimas necesarias a esas personas para su vida cotidiana.

\section{Emigraciones por desesperanza del pueblo venezolano}

En américa latina esta situación ha avanzado en los últimos años posicionando a esta zona en países emigrantes, al respecto Zamora y Gainza (2014), indican:

América Latina registra variaciones demográficas producto del proceso migratorio durante el siglo $\mathrm{xx}$ por causas diversas, pero en las últimas tres décadas de ese siglo los procesos migratorios obedecen fundamentalmente a desequilibrios y crisis económicas que han provocado desempleo, bajos ingresos y la búsqueda de mejores condiciones de vida. (p.73).

Se evidencia una clara participación de la región en los movimientos demográficos y, más en las últimas tres décadas por medio de las situaciones internas de los países latinoamericanos.

En este orden de ideas, uno de los países latinoamericanos que se une a los países con emigrantes en Venezuela y así lo confirma Díaz (2015) en donde "en el año 1992 había venezolanos en menos de 20 países, con apenas unos 30,000 residentes. Ahora hay venezolanos en 94 países de los 193 miembros de la ONU, es un dato dramático"(s/p). 
Esto afirma el aumento desmedido de la cantidad de personas que han salido de Venezuela, de la misma manera afirma que Venezuela fue por muchos años un país de recepción de personas que llegaban de los países europeos y suramericanos por la gran bonanza que tenía el país basada en la renta petrolera y que daba gran estabilidad a los venezolanos.

Así pues, esto no se encontraba en los países europeos ni suramericanos que estaban dentro de una economía rural. Esta situación se generó como una de las causas primordiales fue la inflación que para Díaz (2015): "Solamente entre 2010 y 2014 (último dato disponible), la inflación acumulada en Venezuela ha sido de $413 \%$, mientras que el valor del dólar ante el bolívar en el mercado negro ha subido más de 7,600\%, pasando de Bs 8,17 a Bs 630\%. (s/p). Estos datos son base para que una economía no sea estable y afecte de una u otra manera la calidad de vida de los venezolanos ya que por consiguiente se van afectar todas las organizaciones y todos los rubros atacando a los ciudadanos que son los más vulnerables en este sentido, no pudiendo controlar sus gastos a causa que no hay una estabilidad en los precios de los productos, tal como lo afirma en su investigación (Treuke, 2016).

Esta situación que se ha venido acrecentando en Venezuela está afectando a todos los sectores de la población, incluso uno de los más afectados son los docentes universitarios, que desde un tiempo siendo uno de los profesionales que brindan formación a todas las áreas del conocimiento y generan aportes por medio de sus prácticas pedagógicas se vieron golpeados por su cada vez menor poder adquisitivo, afectando directamente a sus familias, para Gillespie (2017), establece. 'Una gran mayoría de profesores se han ido buscando una mejor vida en otros países mientras Venezuela se sumerge en una profunda crisis humanitaria y económica, marcada por un gobierno que muchos, incluyendo el presidente Donald Trump, consideran una dictadura" (s/p). de esta manera la autora señala que se están perdiendo miles de puestos de trabajos en las universidades venezolanas por estas situaciones señaladas, esto convirtiéndose en un éxodo masivo de profesionales universitarios en busca de nuevas oportunidades afectando con ello la calidad de la educación superior a futuro en el país (Gamboa-Suárez, 2016).

Así mismo, para el Nacional (2017): "Según el presidente de la Asociación de Profesores Universitarios, entre $30 \%$ y $50 \%$ de los catedráticos que ocupan cargos académicos en las referidas casas de estudio se está marchando de Venezuela. No les alcanza el sueldo para la atención de sus necesidades más elementales" (s/p). Con esto se evidencia que los docentes universitarios no pueden cubrir sus necesidades básicas y por ende no pueden mantener a sus familias dentro de Venezuela, siendo una de las principales razones de su emigración, y los datos son alarmantes cuando se ve que llegan al 50\% el éxodo de docentes de las diferentes casas de estudios de educación superior, aunado a esto las situaciones y ambientes de trabajo ha desmejorado por cuanto los presupuestos anuales de las diferentes casas de estudio el gobierno las ha mermado es más del $60 \%$ y las condiciones laborales bajaron significativamente afectado así a los docentes.

Ante esto los docentes enfrentas situaciones complejas, y así lo refleja el Nacional (2017): "La estrechez económica les impide la adquisición de la bibliografía que necesitan para estar al día. El cheque quincenal ni siquiera les alcanza para los gastos de transporte". (s/p). esta difícil situación a nivel humanitaria obliga a los venezolanos a salir de su país de origen el cual los vio nacer, y va aumentando las situaciones que afectan a los venezolanos por cuanto la Acnur (agencia para refugiados de la Onu), estableció como refugiados a los venezolanos que salen de sus fronteras llegando a niveles precarios todas las dimensiones de la vía de los habitantes teniendo problemas hasta para la alimentación básica de los integrantes de las familias, y por consiguiente la de los profesores universitarios que forman parte de la sociedad activa de Venezuela.

De esta manera los docentes universitarios del estado Táchira viven esta misma situación en donde las principales universidades del estado como la Universidad Experimental del Táchira, la 
Universidad de los Andes y Universidad Católica del Táchira y otras de menor envergadura están pasando por esta situación del éxodo docente, es por ello que se estableció como población de estudio para los informantes claves los docentes de la Universidad Nacional del Táchira, según Camargo (2017) señalo: "Se refirió a la replanificación del semestre, en este sentido indicó que se adelantan reuniones continuas tanto con el sector estudiantil como el docente para llegar algunas decisiones en consenso que permitan activar en un $100 \%$ la universidad". ( $\mathrm{s} / \mathrm{p})$, con esto se evidencia la situación de la casa de estudios Unet sobre las consecuencias detalladas anteriormente, de la misma manera Jaimes (2017) indica: "Según el rector, son múltiples las causas del éxodo en la UNET, pero de manera específica el factor económico es primordial al momento de decidir dejar la universidad, las condiciones sociales del país también influyen" ( $\mathrm{s} / \mathrm{p})$. Esto evidenciando de igual manera la situación del éxodo de la universidad.

Es por ello y la creciente preocupación mundial que tiene la mirada en américa latina y específicamente en Venezuela que se plantea esta investigación dirigida a estudios de casos específicos de algunos profesionales universitarios y ver su contexto y experiencias basados en el fenómeno que los abarca sobre las motivaciones de salir de sus países de, origen y empezar un nuevo camino en los países receptores.

Ante esta situación se plantean los siguientes objetivos de investigación.

En la presente investigación se estableció como objetivo general: Analizar la movilidad de docentes universitarios de la universidad Experimental del Táchira dentro de la complejidad de los escenarios contemporáneos. Así mismo como objetivos específicos:

1. Explorar la situación documentada de la movilidad de los docentes universitarios de la Unet en la contemporaneidad.
2. Describir los contextos que maneja la Universidad sobre la movilidad de los docentes de la Unet en los últimos dos años.

3. Develar por medio de un estudio de casos las motivaciones de los docentes movilizados hacia los países latinos.

\section{Migración}

En la presente investigación se sustenta por medio de las conceptualizaciones para seguir entendiendo el escenario descrito, es por ello que se puede definir la migración como:

En este sentido para la universidad Autónoma de México, en su portal sobre movimientos de la población la define como el desplazamiento que realiza una persona o un grupo de personas para cambiar su lugar de residencia, ya sea de un país a otro, o dentro del mismo país.

Asimismo, la Cepal (Comisión Económica para América Latina), define un tipo de migración interna, siendo un componente decisivo de los procesos de redistribución espacial de la población y tiene implicaciones para comunidades, hogares y personas. Para las comunidades, tiene efectos demográficos, sociales, culturales y económicos. Para los hogares y las personas, la migración, en particular si se enmarca en una estrategia elaborada, es un recurso para el logro de determinados objetivos, los que pueden ser tan variados como enfrentar una crisis económica o mejorar la calidad de vida. Los censos son la principal fuente de información sobre estos flujos.

\section{Migraciones según su escala geográfica}

Para el portal Ayuda en Acción (2018), definen que las migraciones externas o internacionales, son las más comunes, que son un proceso clave para la redistribución de oportunidades para sus ciudadanos. También presentan la migración interna siendo un tipo de migración humana mucho menos controlada que la externa, pues los ciudadanos de 
un país tienen, en la mayoría de los casos, derecho a residir en cualquier provincia o ciudad del territorio; por el contrario, la migración internacional queda siempre sujeta a una doble legislación impuesta por el país de origen y el país de destino. Si el migrante cumple la ley, decimos que es un inmigrante legal, mientras que, si no lo hace, se le denomina ilegal: muchas personas están en desacuerdo con este calificativo, alegando que las personas no pueden ser ilegales, y defendiendo el uso de otros términos, como irregular o indocumentado.

\section{Migración Irregular}

Otro tipo de migración que se presenta es la irregular, señalada por Castles (2010), en donde los migrantes irregulares están inmersos en la flexibilización del mercado laboral, así mismo el autor señala sobre un elemento central de la globalización neoliberal pudiendo ser contratados $\mathrm{y}$ despedidos fácilmente, sin procesos complejos o altos costos para el patrón. Con frecuencia se puede dividir los procesos de producción en la manufactura, de modo que las actividades de alta productividad y alta capacitación pueden realizarse en las economías avanzadas, mientras que los segmentos de baja productividad y bajos salarios pueden ser maquilados.

\section{Materiales y métodos}

En el contexto de esta investigación se basa bajo un enfoque cualitativo por cuanto se caracterizará las experiencias de los informantes claves que revelan sus experiencias basadas en sus vivencias, por su parte Hecheverria (2005), señala:

"El análisis cualitativo busca conocer el significado que está inmerso en la trama tejida por el texto o discurso de los sujetos entrevistados. Entonces, se hace necesario situarse desde el punto de mirada del otro, para trabajar de manera inductiva a partir de los datos recogidos. De esta forma se busca partir de lo particular, sumando varios particulares, para ir a un nivel mayor de inteligibilidad".
Es por ello que se estableció el presente enfoque, esto permite sacar información valiosa basada en las experiencias de los informantes claves que son los docentes universitarios escogidos para la investigación.

Asimismo, se sustentará bajo un diseño de campo y descriptivo ya que se recogerá de los informantes claves las experiencias basadas en sus vivencias, de este mismo modo se describirá las experiencias de cada uno de ellos a fin de develar el fenómeno a estudiar basado sobre las motivaciones que tuvieron los docentes universitarios venezolanos en cuanto a la emigración desde sus países de origen.

La población de la presente investigación se trató sobre los docentes universitarios de la universidad Nacional del Táchira que han salido de Venezuela que se encontraban trabajando por más de diez años en la principal universidad del occidente de Venezuela.

De esta manera se procedió a utilizar una muestra intencional por cuanto el universo es amplio y se escogerá aleatoria mente bajo características similares, a su vez Otzen y Manterola (2017), señala sobre las muestras aleatorias: Permite seleccionar casos característicos de una población limitando la muestra sólo a estos casos. Se utiliza en escenarios en las que la población es muy variable y consiguientemente la muestra es muy pequeña. En este sentido la muestra que se seleccionó fueron cinco docentes de la Unet que se encuentras en diferentes países siendo los destinos más buscados de Latinoamérica como Colombia, Ecuador, Perú, Argentina y Chile.

Por consiguiente, la población es de 350 docentes que, según una entrevista informal con el rector de la universidad, estos docentes han salido los que han renunciado por salir del país. 


\section{Resultados y discusiones}

Tabla 1. Respuestas dada por docentes de diferentes paises

\begin{tabular}{|c|c|c|c|}
\hline Informante & Pregunta & Respuesta & Categorías \\
\hline $\begin{array}{c}\text { Asesor jurídico de la } \\
\text { Universidad Nacional } \\
\text { Experimental del Táchira }\end{array}$ & $\begin{array}{c}\text { ¿Qué información maneja } \\
\text { en cuanto a la renuncia } \\
\text { progresiva de los } \\
\text { docentes de la } \\
\text { Universidad durante el } \\
\text { periodo } 2015 \text { - } 2017 ?\end{array}$ & $\begin{array}{l}\text { Respuesta: la salida ya } \\
\text { tiene tiempo dándose por } \\
\text { parte de los docente que } \\
\text { por diferentes motivos } \\
\text { que señalan en sus } \\
\text { escritos se presencia } \\
\text { permisos no remunerados } \\
\text { que son parte de sus } \\
\text { beneficios, permisos para } \\
\text { estudio en el exterior, } \\
\text { permisos no remunerados } \\
\text { para algún tipo de } \\
\text { investigación, ellos no } \\
\text { presentan como tal una } \\
\text { carta de renuncia sino } \\
\text { que agotan las instancias } \\
\text { superiores y salir del país } \\
\text { por tiempo pensando que } \\
\text { la situación en Venezuela } \\
\text { se va arreglar, sin } \\
\text { embargo la información } \\
\text { que manejamos es } \\
\text { que por derecho pueden } \\
\text { pero el motivo de la } \\
\text { salida del país es otro, y } \\
\text { basado en sus } \\
\text { necesidades. } \\
\text { ellos no la reflejan en sus } \\
\text { solicitudes, y es que se } \\
\text { van del país porque las } \\
\text { situaciones que están } \\
\text { viviendo los están } \\
\text { delictivo en la ciudad, } \\
\text { afectando directamente } \\
\text { por no poder cubrir las } \\
\text { necesidades mínimas } \\
\text { necesarias para la } \\
\text { subsistencia de sus } \\
\text { familias, en salud, } \\
\text { alimentación, educación } \\
\text { importantes las más } \\
\text { sus familias ya que se } \\
\text { entidad } \\
\text { anto el alto índice }\end{array}$ & $\begin{array}{l}\text { Necesidades Básicas } \\
\text { Alimentación } \\
\text { Salud } \\
\text { Seguridad }\end{array}$ \\
\hline
\end{tabular}


N. 01 Docente en Colombia

\section{3. ¿Cómo ve la situación de Venezuela?}

1. ¿Qué lo motivo a salir de Venezuela teniendo un trabajo estable en una universidad Nacional y que fecha salió?

2. ¿Por qué escogió Colombia como destino?

1. ¿Qué lo motivo a salir de Venezuela teniendo un trabajo estable en una universidad Nacional y que fecha salió?
1. Respuesta: Una de las principales razones por la que salí del país fue la situación compleja que nos agarró siendo docentes universitarios con más de 14 años de experiencia, por eso me fui en el 2015, ya que poco a poco fue bajando el poder adquisitivo y la calidad de vida que yo llevaba no se podía mantener

2. Respuesta: el motivo porque escogí Colombia fue por mi situación ya que mi padre es de origen colombiano y procedí a tramitar mí cedula colombiana para adquirir mi nacionalidad, y con eso tuve más oportunidades de quedar trabajando en una universidad.

3. Respuesta: La situación es cada vez más compleja ya que el salario de los docentes universitario es afectado directamente por la hiperinflación y nosotros no tenemos los beneficios del gobierno en cuanto a los subsidios de alimentos importados y prácticamente no alcanza para comer mucho menos para vivir.

1. Respuesta: me fui de Venezuela en el 2016 y las principales motivaciones de mi salida de Venezuela se apegan desde que tuve un percance con un familiar y no podía solucionar la situación ya que no conseguí medicinas estando ella en la clínica, esta situación me afecto 
N. 02 Ecuador

1. ¿Qué lo motivo a salir de Venezuela teniendo un trabajo estable en una universidad Nacional y cuando se fue?

2. ¿Por qué escogió Ecuador como destino?

3. ¿Cómo ve la situación de Venezuela?

de tal manera que quede imposibilitado en poder hacer algo y donde entra en juego la vida de las personas e la familia, de la misma manera la situación económica del país afecto los ingresos no son suficientes para cubrir los gastos mínimos para vivir cómodamente.

\section{Respuesta: Me vine a} Ecuador porque es una economía dolarizada y el dólar es una moneda fuerte a nivel mundial, además que la calidad de vida según otros

compañeros es mejor y lo he podido comprobar, porque aquí vivo bien con un sueldo de docente.

3. Respuesta: bastante compleja ya que no se ve que tenga un futuro próspero, los antecedentes dicen que de seguir el gobierno y sus políticas jamás saldrá adelante, la economía cada vez se ve afectada por las políticas emanadas del gobierno.

1. Respuesta: la situación en Venezuela ha evolucionado perjudicialmente a tal punto que un trabajo digno como un docente universitario no puede vivir cómodamente, es por ello que Salí de mi país en el 2015, ya que ni si quiera con una esposa también universitaria, sumando los dos sueldos no se puede cubrir para los gastos mensuales, aunado a eso los gastos de los hijos para cubrir
Salud

Familia

Seguridad

Políticas económicas 
N.03 Perú

2. ¿Por qué escogió Perú como destino?

3. ¿Cómo ve la situación de Venezuela? una buena salud y seguridad ante un percance. Tal situación nos afectó mucho donde se tuvo que vender un carro para reparar el otro, vender prendas para poder subsistir para la Salario comida y entre otras situaciones también la inseguridad era permanente y no podía Salud estar ni física ni mentalmente tranquilo.

2. Respuesta: Perú es un país que ve emergiendo económicamente y por familiares que ya habían llegado aquí tome la decisión de venirme, esto nos ayudó a tener a donde llegar siendo la posición más importante para tomar la decisión.

Respuesta: la situación de mi país la veo muy delicada porque cada día más se ve el desmoronamiento el sistema económico llevando a la hiperinflación y esto nos afecta a todos.

sentido para buscar algo de comida siempre se tenía que hacer cola a esperar que llegara la comida, y esto se presentaba en todos los establecimiento o a veces pasaban meses y no llegaban los productos, de la misma manera pasaba con los medicamentos no se encontraban medicamentos y se tenía que ir a la frontera con Colombia para poderlos comprar, pero con un
Cesta básica 
N.04 Argentina

2. ¿Por qué escogió

Argentina como destino?

3. ¿Cómo ve la situación de Venezuela?

1. ¿Qué lo motivo a salir de Venezuela teniendo un trabajo estable en una universidad Nacional? sueldo de 5 dólares mensuales uno no le alcanzaba para poderlos

Escases de productos adquirir, esta situación

llevo por más de dos años hasta que llego el punto y no pude aguantar más, y tome la decisión de irme.

2. Respuesta: Me fui a la Argentina porque fuimos un grupo de profesores de varias universidades como la Upel, y la Católica que tomamos la decisión de irnos acompañados por carreta, como uno de los destino que estaba apoyando a los venezolanos para poder estar legalmente.

3. Respuesta: la situación de Venezuela la veo muy complicada y no tiene arreglo por lo menos por estos 20 años, $\mathrm{y}$ esto le depara un fututo a los venezolanos incierto y desolador.

\section{Respuesta: las} decisiones para salir de Venezuela no son fáciles, ya que nosotros teníamos todo una comodidad y una estabilidad económica, no teníamos necesidad de ninguna clase, sin embargo la situación fue incrementándose y el poder adquisitivo fue deduciéndose cada vez más llegando al punto de no poder mantener la vivienda, carros, ni siquiera la comida para el mes afectando así a toda la familia, esta situación abarco a todos los integrantes de la familia hasta los hijos que estaban terminando sus
Salario 


\section{N.05 Chile}

\section{3. ¿Cómo ve la situación} de Venezuela?
2. ¿Por qué escogió Chile como destino?

carreras académicas.

Teniendo la motivación de seguir con una buena calidad de vida se tomó la decisión de emigrar.

2. Respuesta: Se prefirió chile por ser la primera economía emergente en Suramérica, teniendo una de las calidades de vida más evidente y para tomar la salida del país pende irme a uno de los mejores países de Latinoamérica, chile a nivel académico también ha evolucionado teniendo una de los modelos educacionales más llamativos.

3. Respuesta: la situación de Venezuela la veo muy grave ya que no se aprecia algún signo esperanza, y la economía va para abajo y esto lo que hace es que más venezolanos se vallan de su país, y una situación que lamentablemente no cambiara hasta que el gobierno de valla.

\section{Categorización}

Entre las categorías encontradas de los estudios descriptivos de los docentes entrevistados se tienen las siguientes:

Tabla 2. Categorías de estudio

\begin{tabular}{ll}
\hline Categorías & \multicolumn{1}{c}{ Análisis } \\
\hline Dentro de las categorías emergentes se tienen que los \\
docentes no cuentan con una seguridad a nivel de la \\
salud para ellos o sus familiares, esto teniendo en \\
cuenta la grave situación que las unidades médicas no \\
cuentan con insumos médicos ni medicamentos, por \\
cuanto teniendo un seguro que cubre todo las clínicas \\
no cuentan con medicinas para poderlas proporcionar \\
y tienen que salir a buscarlas en otros lados incluso a \\
los países vecinos, la situación se complica cuando \\
esas medicinas están fuera de Venezuela en dólares y \\
los ingresos de los docentes no están a nivel de los \\
sueldos internacionales al contrario están bajo casi un
\end{tabular}


Salario (poder adquisitivo)

Necesidades básicas (escases de productos)

Inseguridad

Políticas económicas
$80 \%$ de los sueldos de los docentes promedio en

Latinoamérica, y de ninguna manera se puede conseguir alguna seguridad a nivel de salud.

Así mismo el salario de los docentes que venía decreciendo poco a poco en los últimos siete años en estos dos años supero los índices porque bajo a tal punto que el sueldo docente no alcanzaba para cubrir ni si quiera la cesta básica, tampoco la parte médica, seguridad para tener económicamente una estabilidad y mantener un carro o la vivienda, llegando a niveles que con un sueldo para lo único que puede cubrir a nivel de alimentación es la de cuatro días, ya de ahí hacia adelante es imposible sustentar los requerimientos mínimos necesarios para la comida, y de allí las demás situaciones que llevan una vida cotidiana normal.

Aparte de la situación de los ingresos por medio del salario del docente otra que aparece es la escases de productos ya que no se consiguen los rubros de la cesta básica, y cuando llegan o aparecen se tiene que hacer una cola de por lo menos medio día para solo conseguir un rubro, y de allí salir a buscar en otro lado donde pueden conseguir los demás productos, y llegando al nivel que los productos desaparecen hasta por seis meses o más.

Otro factor importante develado por los docentes fue la inseguridad que agobia a los venezolanos por cuanto no pueden vivir de manera tranquila porque los índices de inseguridad se fueron acrecentando cada vez más, afectando a cualquier sector de la sociedad, llegando a niveles que los propios organismos de seguridad tienen intérnenmele personas encargadas de ser delincuentes, esto conlleva a que no existe un estado de derecho y una impunidad que desampara alguna posibilidad de esperanza de los docentes, y de la misma manera pensando que algún día pudieran ser afectados algún familiar a causa de la delincuencia en la ciudad. Esto es una de las mayores preocupaciones de los docentes de la Unet para tomar la decisión de salir.

En este contexto los docentes establecen sobre las políticas económicas que han implementado en Venezuela en donde progresivamente ha aumentado el poco rendimiento de los salarios de los docentes de

la universidad, y cada vez se sigue agudizando la situación, ya que estas políticas direccionan hacia el bloqueo de la demanda y oferta como ley libre mundial económica y amarra a que todo lo maneja el gobierno y teniendo como consecuencia el cierre de esas organizaciones, de tal manera que las políticas

Fuente: El Investigador

Se deja claro los diferentes escenarios que motivaron a los docentes de esta casa de estudios superiores a tomar la decisión de partir de su país de origen, teniendo durante muchos años establecido todo su comodidad y estilo de vida, esto a su vez afectando a la universidad dejando vacíos académicos en donde la institución ha apoyado los procesos formativos, y de una u otra manera para avanzar y mejorar hacia la calidad y de repente se presenta una deserción docente como se pudo analizar en la categorización anterior.

Como se pudo develar en la descripción de las categorías el factor salud es una dimensión esencial para todo trabajador y especialmente para los docentes siendo formadores profesionales, por su parte Bermejo (2016) señala:

"El trabajo del docente se enmarca dentro de lo que conocemos como profesiones de servicios, y dentro de éstas, los profesores somos empleados de servicios humanos. Esto implica que en nuestro día a día tenemos que trabajar con y para otras personas, fundamentalmente los alumnos, pero también con los padres, el resto del equipo docente, etc". (p.02).

Como lo indica la autora se puede inferir la importancia de aquellas personas prestadoras de servicios, y que tendrá a su cargo atender personas y en la educación son los estudiantes que reciban esa atención 
por medio de los conocimientos que deben recibir y generar, y para esto se debe tener condiciones idóneas para poder realizar las actividades los más optimas posibles.

Para Restrepo y otros (2013), establecen que el bienestar del docente universitario se puede estudiar desde cuatro enfoques, siendo uno ecologista la relación del entorno y el docente, también una dimensión económica la cual fue señalada por los docentes como una de las categorías emergentes que más se acentuó como factor para la movilidad de estos, otro una dimensión sociológica en donde estos docentes están siendo afectados por muchas variables a su alrededor que los afecta como personas principalmente, esto afectando su desempeño en el proceso de enseñanza aprendizaje teniendo como consecuencias la deserción laboral.

Ante esto debe existir un clima motivacional en los salones de clase, las cuales Leal (2014), establece dentro de las teorías de la motivación escolar, centrándose este en el compromiso, persistencia, y desempeño, en donde actualmente esto se está afectando para estos docentes universitarios. Para el docente como todo profesional un factor importante en su desempeño es la motivación y Hué (2012), establece que para la motivación el enemigo más importante es la postergación, para el caso de estos docentes es algo que persiste ya que en su entorno deben postergar la salud, seguridad, economía, siendo esto entre las categorías emergentes factores esenciales para tomar la decisión de salir de su país.

\section{Conclusiones}

En la descripción desde el punto de vista de los distintos docentes de la universidad Nacional Experimental del Táchira que han salido y están establecidos en diferentes países como Colombia, Ecuador, Perú, argentina y Chile y por medio del instrumento aplicado a cada uno de ellos dentro de una entrevista abierta se buscó la categorización de la información en donde se describieron aspectos relevantes para cumplir el objetivo de la presente investigación sobre cuál fue la motivación de la salida de Venezuela, y cada uno de ellos describieron desde sus perspectivas dichas situaciones.

Una de los contextos más resaltantes que describen los docentes sobre esas motivaciones fue la situación para mantener o cubrir las necesidades mínimas necesarias la calidad de vida que venían llevando en la ciudad, por cuanto no podían cubrir una cesta básica completa, ni si quiera con dos sueldos como docentes, aunado a esto las demás situaciones que afectan a los docentes como la inseguridad que ven siempre alguien afectado de su entorno familiar y queda el miedo constante que le pueda suceder a un integrante de la familia, así como también las políticas económicas que han llevado a la grave situación económica a Venezuela, y a pesar de que el gobierno toma acciones cada cierto tiempo ninguna de estas ha servido para congelar la hiperinflación que afecta a los docentes venezolanos, todas estas situaciones integradas son las categorías más relevantes que describen para tomar la decisión de emigrar de Venezuela, afectando así a las casas de estudio y principalmente a la Unet como universidad sede de los docentes entrevistados.

Se recomienda, al Estado venezolano, para que reconsidere su modelo político y obviamente las políticas sociales y económicas; a las autoridades y organismos venezolanos para que implementen programas y propuestas que contengan estratégicas que permitan dar soluciones efectivas a las problemáticas del pueblo venezolano; a los funcionarios de migración y fronteras de Venezuela, se les recomienda asesorarse en materia migratoria y ellos a su vez asesorar al gobierno o a quienes competa para que se tomen en cuenta enfoques, criterios, métodos e instrumentos que permitan mejorar las condiciones de vida de los venezolanos y así evitar la migración masiva actual .

\section{Bibliografía}

Asakura H (2014). Dinámicas interpersonales en el servicio doméstico: el caso de mujeres migrantes centroamericanas en el área metropolitana de Monterrey. Recuperado 
de: http://espacialidades.cua.uam.mx/ vol/05/2015/01/01_Asakura.php

Bermejo L. (2016). Bienestar docente Estrategias para una vida emocionalmente más saludable en el trabajo del profesor.RevistaUPcomillas. Recuperado de: https://revistas.upcomillas. es/index.php/padresymaestros/article/ viewFile/7517/7341

Camargo D (2017). Se trabaja en replanificar el semestre de la Unet. Recuperado de: http://www.unet.edu.ve/eventos-y-noticiasexternas/4203-se-trabaja-en-replanificar-elsemestre-en-la-unet.html

Castles S (2010). Migración irregular: causas, tipos y dimensiones regionales. Revista Migración y desarrollo. Recuperado de: http:// www.scielo.org. $\mathrm{mx} / \mathrm{scielo} . \mathrm{php}$ ? script $=$ sci_ arttext\&pid=S1870-75992010000200002

CEPAL. Migración interna. Recuperado de: https:// www.cepal.org/es/temas/migracion-interna

Díaz (2014). La Emigración crece como plan de vida de los venezolanos. Recuperado de: http:// www.elnuevoherald.com/noticias/mundo/ america-latina/venezuela-es/article27854107. html

Echeverría G (2005). Análisis cualitativos por categorías. Recuperado de: https:// s 3 . amazonaws.com/academia.edu. documents/39046623/ AN ALISIS CUALITATIVO_G_ECHEVERRIA_1.pdf?A WSAccessKeyId=AKIAIWOWYYGZ2Y53U L3A\&Expires $=1523997109 \&$ Signature $=p 7 H L$ d1Nu5wk\%2FjyIo2M2ZF\%2BmgK0Y\%3D\& response-content-disposition $=$ inline $\% 3 \mathrm{~B} \% 20$ f i 1 e $n$ a m e $\% 3$ D A N A L I S I S CUALITATIVO_G_ECHEVERRIA_1.pdf

Hué G. (2012). Bienestar docente y pensamiento emocional. Universidad de Zaragoza. Recuperado: http://institucional.us.es/revistas/ fuente/12/art_1.pdf

El Nacional (2017). El adiós de los profesores. Recuperado de: http://www.el-nacional.com/ noticias/editorial/adios-los-profesores_204879
Gamboa-Suárez, A. A. (2016). Docencia, investigación y gestión: Reflexiones sobre su papel en la calidad de la educación superior. Revista Perspectivas, 1(1), 81-90.

Gómez J. (2010) La migración internacional: teorías y enfoques, una mirada actual., recuperado de: http://www. scielo.org.co/scielo.php? script $=$ sci arttext\&pid=S0120-63462010000100005

Gillespie (2017). Venezuela está perdiendo a sus profesores en medio de un éxodo masivo. Recuperado de:

http:/cnnespanol.cnn.com/2017/12/04/venezuelamigracion-profesores-crisis-universidades/

Jaimes D. (2007). Deserción en la Unet supera los 1.200 estudiantes en 2017. Recuperado de: http://ecosdeltorbes.net/desercion-en-la-unetsupera-los-1-200-estudiantes-en-2017/

Leal F, Ramiez J, Valdivia Y.(2014). Bienestar psicológico y prácticas docentes con efectos motivacionales orientadas al aprendizaje. Universitas Psychologica, 13(3). http:// dx.doi. org/10.11144/Javeriana.UPSY13-3.bppd

Otzen y Manterola (2017). Técnicas de Muestreo sobre una Población a Estudio https:// scielo.conicyt.cl/scielo.php? pid=S071795022017000100037\&script $=$ sci_abstract

Restrepo E, Lopez A, (2013) Percepciones del entorno laboral de los profesores universitarios en un contexto de reorganización flexible del trabajo. Cuadernos de Administración. Recuperado: http://www.scielo.org.co/pdf/ cuadm/v29n49/v29n49a07.pdf

Treuke, S. (2016). El impacto del vecindario en la movilidad económica: examinando las redes sociales de habitantes de tres barrios segregados de Salvador da Bahia (Brasil) a partir del concepto social isolation. Revista Perspectivas, l(1), 6-29.

Universidad autónoma de México. Movimientos de la población. Recuperado de: http://www. objetos.unam.mx/geografia/migraciones/ index.html 
Walteros A. (2010) la migración internacional: teorías y enfoques, una mirada actual. Recuperado de: http://www.redalyc.org/ pdf/1650/165014341004.pdf

Zamora y Gainza (2014). Economía, migración y política migratoria en Sudamérica: Avances y desafíos. Recuperado de:

http://www.redalyc.org/pdf/660/66038803003.pdf 